\title{
Skin picking disorder: prevalence, correlates, and associations with quality of life in a large sample
}

\author{
Myrela O. Machado, 'Cristiano A. Köhler, ${ }^{\prime}$ Brendon Stubbs, ${ }^{2,3,4}$ \\ Paulo R. Nunes-Neto, ${ }^{1}$ Ai Koyanagi, ${ }^{5}$ João Quevedo, ${ }^{6,7,8,9}$ \\ Jair C. Soares, ${ }^{8}$ Thomas N. Hyphantis, ${ }^{10}$ Donatella Marazziti, ${ }^{11}$ \\ Michael Maes, ${ }^{12,13}$ Dan J. Stein, ${ }^{14}$ and André F. Carvalho ${ }^{1,15,16 *}$
}

\footnotetext{
${ }^{1}$ Department of Clinical Medicine and Translational Psychiatry Research Group, Faculty of Medicine, Federal University of Ceará, Fortaleza, CE, Brazil

${ }^{2}$ South London and Maudsley NHS Foundation Trust, Denmark Hill, London, UK

${ }^{3}$ Institute of Psychiatry, Psychology and Neuroscience (IoPPN), King's College London, London, United Kingdom

${ }^{4}$ Faculty of Health, Social Care and Education, Anglia Ruskin University, Chelmsford, UK

${ }^{5}$ Parc Sanitari Sant Joan de Déu, Universitat de Barcelona, Fundació Sant Joan de Déu/CIBERSAM, Barcelona, Spain

${ }^{6}$ Translational Psychiatry Program, Department of Psychiatry and Behavioral Sciences, McGovern Medical School, The University of Texas Health Science Center at Houston (UTHealth), Houston, Texas, USA

${ }^{7}$ Laboratory of Neurosciences, Graduate Program in Health Sciences, Health Sciences Unit, University of Southern Santa Catarina (UNESC), Criciúma, SC, Brazil

${ }^{8}$ Center of Excellence on Mood Disorders, Department of Psychiatry and Behavioral Sciences, McGovern Medical School, The University of Texas Health Science Center at Houston (UTHealth), Houston, Texas, USA

${ }^{9}$ Neuroscience Graduate Program, The University of Texas MD Anderson Cancer Center UTHealth Graduate School of Biomedical Sciences, Houston, Texas, USA

${ }^{10}$ Department of Psychiatry, Faculty of Medicine, School of Health Sciences, University of Ioannina, Greece

${ }^{11}$ Department of Clinical and Experimental Medicine, Section of Psychiatry, University of Pisa, Pisa, Italy

12 Department of Psychiatry, Faculty of Medicine, Chulalongkorn University, Bangkok, Thailand

${ }^{13}$ IMPACT Research Center, Deakin University, Geelong, Australia

${ }^{14}$ Department of Psychiatry and MRC Unit on Risk \& Resilience in Mental Disorders, University of Cape Town, Cape Town, South Africa

${ }^{15}$ Department of Psychiatry, University of Toronto, Toronto, Ontario, Canada

${ }^{16}$ Centre for Addiction \& Mental Health (CAMH), Toronto, Ontario, Canada
}

Objective. Evidence suggests that skin picking disorder (SPD) could be a prevalent condition associated with comorbidity and psychosocial dysfunction. However, just a few studies have assessed the prevalence and correlates of SPD in samples from low- and middle-income countries. In addition, the impact of SPD on quality of life (QoL) dimension after multivariable adjustment to potential confounders remains unclear.

Methods. Data were obtained from a Brazilian anonymous Web-based research platform. Participants provided sociodemographic data and completed the modified Skin Picking-Stanford questionnaire, the Hypomania Checklist (HCL-32), the Patient Health Questionnaire-9 (PHQ-9), the Fagerström Test for Nicotine Dependence, Alcohol Use Disorder Identification Test (AUDIT), Symptom Checklist-90-Revised inventory (SCL-90R), early trauma inventory self report-short form, and the World Health Organization quality of life abbreviated scale (WHOQOL-Bref). Associations were adjusted to potential confounders through multivariable models.

Results. For our survey, 7639 participants took part (71.3\% females; age: $27.2 \pm 7.9$ years). The prevalence of SPD was $3.4 \%$ (95\% CI: $3.0-3.8 \%$ ), with a female preponderance $(P<0.001)$. In addition, SPD was associated with a positive

* Address for correspondence: André F. Carvalho, MD, PhD, Centre for Addiction \& Mental Health, 33 Russel Street, Room RS 1050, Toronto, Ontario, Canada, M5S 2 S1.

(Email: andre.carvalho@camh.ca)

The Translational Psychiatry Program (USA) is funded by the Department of Psychiatry and Behavioral Sciences, McGovern Medical School, The University of Texas Health Science Center at Houston (UTHealth).

Laboratory of Neurosciences (Brazil) is one of the centers of the National Institute for Molecular Medicine (INCT-MM) and one of the members of the Center of Excellence in Applied Neurosciences of Santa Catarina (NENASC). Its research is supported by grants from CNPq, FAPESC; Instituto Cérebro e Mente and UNESC. JQ is a $1 \mathrm{~A} \mathrm{CNPq} \mathrm{Research} \mathrm{Fellow.}$

AFC is supported by a research fellowship award from CNPq. CAK is the recipient of a postdoctoral fellowship award from the Coordenação de Aperfeiçoamento de Pessoal de Nível Superior (CAPES, Brazil). The authors thank Drs. Ives C. Passos, Aristides V. Cordioli, Albina R. Torres, Joel P. Pinto, Ygor A. Ferrão, and Izio Klein for providing expert assessment of the modified Brazilian Portuguese version of the Skin Picking-Stanford Questionnaire. 
screen for a major depressive episode, nicotine dependence, and alcohol dependence, as well as suicidal ideation. Physical and psychological QoL was significantly more impaired in participants with SPD compared to those without SPD, even after adjustment for comorbidity.

Conclusions. In this large sample, SPD was a prevalent condition associated with co-occurring depression, nicotine, and alcohol dependence. In addition, SPD was independently associated with impaired physical and psychological QoL. Public health efforts toward the early recognition and treatment of SPD are warranted.

Received 25 September 2017; Accepted 3 November 2017; First published online 7 May 2018

Key words: Epidemiology, obsessive-compulsive related disorders, psychiatry, quality of life, skin picking disorder.

\section{Introduction}

Skin picking disorder (SPD), also referred to as dermatillomania, psychogenic excoriation, and excoriation disorder, was formally included into the psychiatric classification system as an obsessive-compulsive related disorder (OCRD) in the 5th edition of the Diagnostic and Statistical Manual of Mental Disorders (DSM-5). ${ }^{1-3}$ The inclusion of SPD in the nosology reflected a growing awareness of the prevalence of SPD, as well as its high comorbidity and morbidity. ${ }^{3}$ Nevertheless, many gaps in the literature remain.

First, prevalence rates of SPD markedly varied across studies. In a telephone-based US community survey the prevalence of SPD was $1.4 \%,{ }^{4}$ while $5.4 \%$ of adults reported significant skin picking associated with distress or impact and, therefore, met criteria for SPD in another US nonclinical population study. ${ }^{5}$ In both studies the presence of SPD was defined by means of a self-reported instrument. There are notably few epidemiological studies from low- and middleincome countries (LMICs). ${ }^{6-8}$ Furthermore, these studies enrolled convenience nonclinical samples (eg, university students) and used a self-reported tool to assess SPD.

Second, although SPD has been associated with high rates of co-occurring anxiety, mood, substance use, and other OCRDs, ${ }^{3,9-11}$ as well as with impaired quality of life (QoL), ${ }^{12,13}$ the impact of SPS on QoL dimensions after multivariable adjustment to sociodemographic and clinical covariates (eg, co-occurring mental disorders) remains unclear. Yet the associations of SPD and co-occurring mental disorders and QoL are to a large extent derived from clinical samples, and thus further research in population or nonclinical samples is warranted. Furthermore, in more dramatic clinical situations, SPD may result in significant and even life-threatening medical complications (eg, septicemia), ${ }^{3}$ although the association of SPD and suicidality has not been completely elucidated. Finally, a previous community study reported a significant association between skin picking and exposure to childhood sexual abuse. ${ }^{14}$ A recent small clinical study also found SPD to be associated with exposure to a higher number of traumatic events in childhood, as compared to a control sample. ${ }^{15}$ However, the specificity of this finding deserves further investigation.
Due to the aforementioned gaps in the literature, the current study has 3 aims: (1) to investigate the prevalence of SPD in a large Brazilian sample; (2) to assess sociodemographic and clinical correlates of SPD; and (3) to determine the independent impact of SPD on QoL dimensions.

\section{Methods}

\section{Sample selection}

Consecutive participants $(\mathrm{N}=9,603)$ were recruited through a large Web-based Brazilian study (Portal Temperamento e Saúde Mental, www.temperamentoesaudemental.org), which is a project that aims to investigate the frequency and correlates of several disorders and psychopathological conditions through the use of validated self-reported measures. ${ }^{16,17}$ This Website provides an encrypted and confidential platform for data collection. The research ethics committee of the Hospital Universitário Walter Cantídio (HUWC) approved the procedures for online data collection under the protocol number 1.058.252. To access the surveys, participants were required to be at least 18 years old and to sign a digital informed consent form. Potential participants were individuals living in Brazil who had internet access; no incentives were granted for participation in this survey. Several attention and validation questions throughout the protocol were employed to assess the quality of the data. Those questions included, for example, "How old are you?" and "How much attention are you paying while answering to this survey?" Consistency of responses were verified (ie, participants had previously provided their dates of birth), while participants who indicated that they were not paying adequate attention to the questionnaires were excluded. This exploratory study included participants who had provided valid responses to these questions. From the initial sample, 9,585 participants answered the complete survey. After quality checking, 7,639 subjects remained eligible (ie, provided correct responses to the validation/ attention questions) and were included in the final analyses (response rate: $79.7 \%$ ). There were no statistically significant differences in age, gender distribution, 
and education level between participants who were not included in the final sample compared to those that did not pass our quality check, and hence were included in final analyses (data available upon request).

This online survey collected sociodemographic data (age, sex, educational level, ethnicity, marital status, religious affiliation, occupation, and gross monthly income). In addition, the web-based platform included several validated psychological and psychiatric measures, which are described below.

\section{Measures}

Skin Picking Stanford Questionnaire (SPSQ)

The SPSQ is self-report measure that comprises 13 questions that address the phenomenology of SPD using a yes/no/don't know format. ${ }^{4}$ The authors of the current study decided a priori to eliminate from the original version of the questionnaire the question "Could you write the name of that medical condition?", which refers to a possible underlying medical condition that could explain the skin picking behavior. This question is not essential to the case definition of SPD based on DSM-5 criteria. $^{2}$ However, we maintained the question "Do you pick your skin because it is inflamed or itchy due to a medical condition?", which is consistent with the DSM-5 exclusion criterion. This 12-item modified version of the SPSQ was translated to Brazilian Portuguese, then back translated into English. Three bilingual authors (MOM, CAK, and AFC) compared the back-translated version to the original version of the SPSQ, and modifications to ensure semantic equivalence were performed. This Brazilian Portuguese version of the SPSQ was tested in a pilot sample of 5 outpatients of the psychiatry service of the HUWC who reported no difficulties in understanding items of this instrument. Six experts in the field of OCRDs (see the "Acknowledgments" section of this article) provided a qualitative assessment of the content validity of the SPSQ. In brief, experts were asked to provide comments on each item of the SPSQ regarding grammar, wording, scaling, and item allocation, as well as the accuracy, clarity, style, and relevance of the translation. We calculated the content validity index (CVI) as described in detail elsewhere. ${ }^{18,19}$ To compute the CVI, members of the expert panel were asked to rate each SPSQ item in terms of relevance, clarity, and simplicity on a Likert scale from 1 to 4 ( 1 was the lowest grade in each of these aspects, while 4 was the highest). The CVI for each item was computed as the number of experts assigning a rate of 3 or 4 to the item divided by the total number of experts. The overall SPSQ CVI value was obtained by averaging all items. The overall CVI of the modified Brazilian Portuguese version of the SPSQ was 0.94 (range for individual items: $0.67-1.00$ ), thus supporting its content validity. In the current sample, the modified SPSQ also had adequate internal consistent reliability (Cronbach's alpha coefficient $=0.73 ; 95 \%$ CI: 0.72-0.74). The final modified Brazilian Portuguese version of the SPSQ is provided in the Supplementary Material (available online). A positive screen for SPD was considered when study participants provided affirmative responses to questions 6,7 , and 8 of the SPSQ. In addition, participants had to endorse at least one additional manifestation of SPD (ie, at least 1 affirmative response to questions 3,4 , and 5 of the SPSQ).

\section{Hypomania checklist (HCL-32)}

The HCL-32 consists of 32 yes/no questions, and investigates the presence of a wide range of (hypo) manic symptoms. ${ }^{20}$ Participants were asked to focus on the "high" periods and to indicate whether hypomanic manifestations were present during this state. In addition, the HCL-32 includes 8 severity and functional impact items related to the duration of episodes and to positive and negative consequences over different areas of functioning. We used the validated Brazilian Portuguese version of the HCL-32 with the recommended cutoff of 19 for nonclinical samples. ${ }^{21}$ In addition, for a positive screening for a bipolar spectrum disorder, participants had to endorse an impairment in at least 1 area of functioning due to the presence of hypomanic symptoms. A previous meta-analysis supports the accuracy of the HCL-32 for the screening of bipolar spectrum disorders. ${ }^{22}$ In the current sample, the reliability of the HCL-32 instrument was adequate (Cronbach's alpha= 0.82; 95\% CI: 0.81-0.82).

\section{Patient Health Questionnaire 9 (PHQ-9)}

Depressive symptoms were assessed with the validated Brazilian Portuguese version of the PHQ-9. ${ }^{23}$ The PHQ-9 questionnaire is a self-report instrument that employs the 9 DSM-IV symptom-based criteria for screening of major depressive episodes. ${ }^{24}$ A positive screening for a major depressive episode was established based on an algorithm in accordance with the validation study of the Brazilian Portuguese version of the MDQ, which was performed in the general population. Furthermore, we used question 9 of the PHQ-9 (ie, "Having thoughts that you would be better off dead, or of hurting yourself on at least 2 days over the past 2 weeks") to screen for the presence of suicidal ideation. ${ }^{25}$ The Cronbach's alpha of the PHQ-9 in the current sample was 0.89 (95\% CI: 0.88-0.89).

Fagerström Test for Nicotine Dependence (FTND)

We used the validated Portuguese version of the FTND to screen for the presence of DSM-IV nicotine dependence. $^{26}$ In brief, the FTND is a 6-item self-report questionnaire with scores ranging from 0 to $10 .{ }^{27} \mathrm{~A}$ 
cutoff score of 4 on the FTND was considered as indicative of nicotine dependence in the current study. ${ }^{26}$ The Cronbach's alpha of the FTND in the current sample was 0.74 (95\% CI: 0.71-0.76).

\section{Alcohol Use Disorders Identification Test (AUDIT)}

We used the validated Brazilian Portuguese version of the AUDIT to screen for the presence of alcohol use disorders. ${ }^{28}$ In brief, the AUDIT is a 10-item self-report questionnaire developed by the World Health Organization (WHO) to screen for the presence of alcoholism (formerly referred to as hazardous or harmful alcohol consumption). ${ }^{29}$ A score $\geq 8$ was considered indicative of the presence of an alcohol use disorder. ${ }^{29}$ In the current study, the AUDIT had adequate reliability (Cronbach's alpha $=0.83 ; 95 \%$ CI: $0.82-0.83$ ).

\section{Early Trauma Inventory Self Report-Short Form (ETISR-SF)}

We used the validated Brazilian Portuguese version of the ETISR-SF to assess exposure to early trauma. ${ }^{30}$ This is a self-report instrument that comprises 27 items grouped into 4 dimensions (general trauma, physical abuse, emotional abuse, and sexual abuse). ${ }^{31}$ The ETISR-SF exhibited adequate internal consistency reliability in the current sample (Cronbach's alpha= 0.86; 95\% CI: $0.86-0.87)$.

\section{Symptom Checklist-90-Revised Inventory (SCL-90R)}

We used the Brazilian Portuguese version of the Symptom Checklist-90-Revised inventory (SCL-90R) to assess psychopathological dimensions. ${ }^{32,33}$ Briefly, the SCL-90R is a 90-item, 5-point, Likert-type inventory, which assesses several psychopathological dimensions namely somatization, obsessive-compulsive, interpersonal sensitivity, depression, anxiety, hostility, phobic anxiety, paranoid ideation, and psychoticism. In this study, Cronbach's alpha for the SCL-90R dimensions ranged from 0.79 (95\% CI: 0.79-0.80) for paranoid ideation to 0.92 (95\% CI: 0.91-0.92) for the depression dimension.

World Health Organization Quality of Life instrumentAbbreviated version (WHOQOL-BREF)

We used the validated Brazilian Portuguese version of the WHOQOL-BREF to assess QoL dimension in the current study. ${ }^{34}$ This generic instrument consists of 26 items assessing QoL in four dimensions: physical, psychological, social, and environment QoL. ${ }^{35}$ Each item is rated on a 5-point, Likert-type scale, and scores are transformed on a scale from 0 to 100, with higher scores indicating higher QoL. Cronbach's alpha values were 0.80 (95\% CI: 0.80 0.81), 0.83 (95\% CI: $0.82-0.84), 0.68$ (95\% CI: 0.67-
$0.70)$, and 0.79 (95\% CI: $0.78-0.79)$ for the physical, psychological, social, and environment domains of the WHOQOL-BREF, respectively.

\section{Statistical analysis}

All statistical analyses were carried out by means of SPSS (IBM, US) version 22.0 for Windows. Continuous variables are presented as means \pm standard deviation (SD). The Kolmogorov-Smirnov test was used to assess whether variables displayed a normal distribution. Sociodemographic and psychopathological variables were compared between participants with vs those without SPD. Normally distributed continuous variables were compared using independent samples Student's t-test. Categorical variables were compared using Pearson's chi-square $\left(\chi^{2}\right)$ or Fisher's exact test as appropriate.

The associations of SPD (dependent variable) and a positive screening for MDD, BD, alcohol or tobacco use disorder, trauma, and suicide ideation, as well as SCL-90 R psychopathological domain scores were assessed using separate multivariable logistic regression models. For the association of SPD and psychopathological dimensions, the scores of each SCL-90 domain were entered as continuous independent variables in the model. For the association of SPD with suicidal ideation, the PHQ-9 question 9 response was entered in the model as a categorical variable. For the associations of SPD and trauma domains, the scores of each individual ETISR-SF domain were entered as continuous independent variables. All other independent variables were categorical. All multivariable models were adjusted by age, sex, occupation, previous use of psychotropic drugs, education level, and ethnicity. Multivariable models that assessed the presence of suicidal ideation, as well as exposure to early life trauma, were additionally controlled for the presence of a positive screening for a major depressive episode, bipolar spectrum disorder, nicotine dependence, and alcohol dependence.

The associations of the presence of SPD and each WHOQOL-BREF domain (dependent variables) were analyzed through separate analysis of covariance (ANCOVA) models. Each model was adjusted by age, sex, occupation, family history of mental disorders, previous use of psychotropic drugs, education level, ethnicity, marital status, gross monthly income, presence of a positive screening for a major depressive episode, bipolar spectrum disorder, a positive screen for suicidal ideation, nicotine dependence, and alcoholism. In addition, we estimated effect sizes of statistically significant (independent) associations of a positive screening for SPD and QoL domains with partial eta squared $\left(\eta_{\mathrm{p}}^{2}\right)$; effect sizes were regarded as small, medium, and large when $0.01<\eta_{\mathrm{p}}^{2}<0.06,0.06 \leq \eta_{\mathrm{p}}^{2}$ 
$<0.14$, and $\eta_{\mathrm{p}}{ }^{2} \geq 0.14$, respectively. ${ }^{36}$ In addition, we estimated the internal consistency reliability of each instrument used in the current study through Cronbach's alpha coefficients (and 95\% CIs). Statistical significance was set at an alpha level of 0.05 .

\section{Results}

Sociodemographic characteristics of the sample are shown in Table 1 . The total sample had a mean age of $27.2 \pm 7.9$ years, was predominantly composed of women $(71.3 \%)$, and most of the individuals had at least secondary-level education. The prevalence of probable SPD was $3.4 \%$ (95\% CI: $3.0-3.8 \%$ ). In addition, the prevalence of probable SPD was significantly higher among women, but was not associated with age or different age groups. Furthermore, participants with probable SPD significantly differed from those without SPD regarding occupation and ethnicity. Finally, participants with probable SPD were more likely to have used psychotropic agents (Table 1).

\section{Clinical correlates of SPD}

The presence of probable SPD was significantly associated with a positive screening for a major depressive episode $\left(\mathrm{OR}_{\mathrm{adj}}=1.854\right)$, nicotine dependence $\left(\mathrm{OR}_{\mathrm{adj}}=2.058\right)$, alcoholism $\left(\mathrm{OR}_{\mathrm{adj}}=1.570\right)$, as well as a positive screening for suicidal ideation $\left(\mathrm{OR}_{\mathrm{adj}}=1.427\right)$, but not for bipolar spectrum disorders (Table 2).

The presence of probable SPD was also independently related with the obsessive-compulsive $\left(\mathrm{OR}_{\mathrm{adj}}=1.339\right.$; $P=0.02)$ and hostility $\left(\mathrm{OR}_{\mathrm{adj}}=1.300 ; P<0.01\right)$ dimensions of the SCL-90R. Moreover, SPD was associated with lower scores in the SCL-90R interpersonal sensitivity dimension $\left(\mathrm{OR}_{\mathrm{adj}}=0.759 ; P=0.04\right)$ (Table 2$)$.

Finally, SPD was significantly associated with exposure to general traumas, psychological abuse, and sexual abuse early in life, but not with physical abuse. However, only associations with general traumas $\left(\mathrm{OR}_{\mathrm{adj}}=1.070 ; \quad P=0.03\right)$ and psychological abuse $\left(\mathrm{OR}_{\mathrm{adj}}=1.216 ; P<0.001\right)$ survived multivariable adjustment to co-occurring mental disorders and sociodemographic covariates (Table 2).

\section{Impact of SPD on quality of life domains}

Probable skin picking disorder was significantly associated with impaired physical and psychological QoL (Figure 1), even after adjusting for sociodemographic variables and comorbidity. However, the presence of probable SPD was not significantly associated with social and environment QoL. All ANCOVA models were statistically significant (adjusted $\mathrm{R}^{2}$ values ranged from 0.195 to 0.442 ). Effect sizes for the adjusted associations of a positive screen for SPD and physical $\left(\eta_{\mathrm{p}}{ }^{2}=0.33\right)$ and psychological $\left(\eta_{\mathrm{p}}^{2}=0.44\right)$ QoL domains were large.

\section{Discussion}

To our knowledge, the present study is the largest survey on the prevalence and correlates of SPD conducted to date. The point prevalence of probable SPD in our sample was $3.4 \%$ with a notable preponderance among women. The prevalence estimate here is within the range of previous community studies that were conducted in the US, and where point prevalence varied from $1.2 \%$ to $5.4 \%,{ }^{4,5,37}$ while the prevalence of possible SPD was $7.6 \%$ in sample of Polish students ${ }^{6}$ and $9.0 \%$ in a sample of 3 medical colleges from Karachi (Pakistan). ${ }^{7}$ Possible sources of heterogeneity in prevalence rates across studies deserve further investigation. For example, Keuthen et $a l^{4}$ performed a telephone-based survey and the presence of possible SPD was assessed with the SPSQ, while the Skin Picking Scale was used in another US community study that included a smaller sample who underwent face-to-face interviews. ${ }^{5}$ In addition, settings and populations have varied across studies. For instance, a prevalence of $5.4 \%$ of possible SPD (assessed by means of the SPSQ) was verified in a study that enrolled Israeli Jewish and Arab clinical samples, ${ }^{38}$ while prevalence rates of possible SPD were $7.6 \%$ and $9.0 \%$ among Polish university students and medical students from Karachi (Pakistan) respectively. ${ }^{6,7}$ Thus, differences in tools used to assess the presence of possible SPD as well as differences in sample selection and settings across studies could have contributed to these discrepant findings. Furthermore, although not all studies are consistent, accumulating evidence indicates that SPD is more prevalent among women. ${ }^{3}$ The present data add to the small number of studies that have been thus far conducted in low- and middle-income countries. ${ }^{6-8}$ Our study also explored several clinical correlates associated with SPD with the use of validated self-report instruments, and found that SPD has a detrimental impact on physical and psychological QoL, even when controlling for sociodemographic variables and comorbidity.

\section{Clinical correlates of SPD}

In the current study, SPD was associated with a positive screening for a major depressive episode, nicotine dependence, and alcohol dependence, even after adjustment for potential confounders. Those findings are consistent with studies conducted in clinical samples. For example, although the prevalence of major depressive disorder has varied across studies (from $12.5 \%$ to $48.0 \%),{ }^{3,11}$ the data suggest that depression is more common among patients with SPD than in the general population. In addition, previous studies that enrolled 
TABLE 1. Sociodemographic and psychopathological characteristics of study participants

\begin{tabular}{|c|c|c|c|c|}
\hline Variable & Total $(\mathrm{N}=7,639)$ & $\mathrm{SPD}(\mathrm{N}=259)$ & № SPD $(N=7380)$ & $P$-value \\
\hline Age, years (mean $\pm S D$ ) & $27.2 \pm 7.9$ & $27.8 \pm 8.4$ & $27.1 \pm 7.8$ & $0.214^{c}$ \\
\hline \multicolumn{5}{|l|}{ Age range } \\
\hline $18-21$ years & $1817(23.8)$ & $64(24.7)$ & $1753(23.8)$ & \multirow[t]{4}{*}{0.110} \\
\hline $22-25$ years & $1984(26.0)$ & $61(23.6)$ & $1923(26.1)$ & \\
\hline $26-30$ years & 1769 (23.2) & $49(18.9)$ & $1720(23.3)$ & \\
\hline$>30$ years & $2069(27.1)$ & $85(32.8)$ & $1984(26.9)$ & \\
\hline \multicolumn{5}{|l|}{ Gender } \\
\hline Female & $5446(71.3)$ & $2133(82.2)^{* *}$ & $5233(70.9)^{*}$ & \multirow[t]{2}{*}{$<0.001^{\circ}$} \\
\hline Male & $2193(28.7)$ & $74.4(17.8)^{*}$ & $2147(29.1)^{* *}$ & \\
\hline \multicolumn{5}{|l|}{ Occupation } \\
\hline Employed & $2768(36.2)$ & $88(34.0)$ & $2680(36.3)$ & \multirow[t]{6}{*}{$0.035^{b}$} \\
\hline Unemployed & $2672(35)$ & $78(30.1)$ & $2594(35.1)$ & \\
\hline Housewife & $368(4.8)$ & $14(5.4)$ & $354(4.8)$ & \\
\hline Retired & $36(0.5)$ & $4(1.5)^{*}$ & $32(0.4)^{* *}$ & \\
\hline Self-employed & $757(9.9)$ & $29(11.2)^{*}$ & $728(9.9)^{* *}$ & \\
\hline Other status & $1038(13.6)$ & $46(17.8)^{* *}$ & $992(13.4)^{\star *}$ & \\
\hline \multicolumn{5}{|l|}{ Family history of mental disorders } \\
\hline Yes & 3335 (43.7) & $125(48.3)$ & 3210 (43.5\%) & \multirow[t]{3}{*}{$0.243^{\mathrm{a}}$} \\
\hline No & $4046(53.0)$ & $128(49.4)$ & $3918(53.1)$ & \\
\hline Don't know (adopted child) & $258(3.4)$ & $6(2.3)$ & 252 (3.4) & \\
\hline \multicolumn{5}{|l|}{ Previous use of psychotropic drugs (N, \%) } \\
\hline Yes, not currently & $1177(15.4)$ & $63(24.3)^{*}$ & $1114(15.1)^{\star \star}$ & \multirow[t]{3}{*}{$<0.001^{\mathrm{a}}$} \\
\hline Yes, currently & $808(10.6)$ & $58(22.4)^{*}$ & $750(10.2)^{\text {** }}$ & \\
\hline № & $5654(74.0)$ & $138(53.3)^{* *}$ & $5516(74.7)^{*}$ & \\
\hline \multicolumn{5}{|l|}{ Educational level (N, \%) } \\
\hline Less than primary school & $86(1.1)$ & $5(1.9)$ & $81(1.1)$ & \multirow[t]{4}{*}{$0.285^{\mathrm{b}}$} \\
\hline Primary school & $483(6.3)$ & $21(8.1)$ & $462(6.3)$ & \\
\hline Secondary school & $4721(61.8)$ & $152(58.7)$ & 4569 (61.9) & \\
\hline University degree & $2349(30.8)$ & $81(31.3)$ & $2268(30.7)$ & \\
\hline \multicolumn{5}{|l|}{ Ethnicity (N, \%) } \\
\hline Caucasian & $3459(45.3)$ & $132(51.0)$ & $3327(45.1)$ & \multirow[t]{5}{*}{$0.035^{b}$} \\
\hline African American & $773(10.1)$ & $32(12.4)$ & $741(10.0)$ & \\
\hline Mulatto $^{d}$ & $2958(38.7)$ & $82(31.7)$ & $2876(39.0)$ & \\
\hline Asian & $87(1.1)$ & 0 & $87(1.2)$ & \\
\hline Other & $294(3.8)$ & $13(5.0)$ & $349(4.7)$ & \\
\hline Marital status (N, \%) & & & & \\
\hline Single & $4849(63.5)$ & $156(60.2)$ & $4693(63.6)$ & $0.072^{\mathrm{a}}$ \\
\hline Married & $1021(13.4)$ & $32(12.4)$ & 989 (13.4) & \\
\hline Stable union & 787 (10.3) & $35(13.5)$ & $752(10.2)$ & \\
\hline Divorced/Widow & $481(6.3)$ & $24(9.3)$ & $457(6.2)$ & \\
\hline Other & $501(6.6)$ & $12(4.6)$ & $489(6.6)$ & \\
\hline Religion (N, \%) & & & & \\
\hline Catholic & $2305(30.2)$ & $71(27.4)$ & $2234(30.3)$ & $0.119^{\mathrm{a}}$ \\
\hline Protestant & 1864 (24.2) & $57(22.0)$ & $1807(24.5)$ & \\
\hline Spiritist & $619(8.1)$ & $32(12.4)$ & $587(8.0)$ & \\
\hline Agnostic & $1203(15.7)$ & $42(16.2)$ & $1161(15.7)$ & \\
\hline Other & $1648(21.8)$ & $57(22.0)$ & $1591(21.6)$ & \\
\hline Gross monthly income (N, \%) & & & & \\
\hline Less than 310.00 USD & $2806(36.7)$ & $93(35.9)$ & $2713(36.8)$ & $0.573^{\mathrm{a}}$ \\
\hline Between 310.00 USD and 931.00 USD & 2549 (33.4) & $90(34.7)$ & $2459(33.3)$ & \\
\hline Between 931.00 USD and $1,863.00$ USD & $736(9.6)$ & $31(12.0)$ & $705(9.6)$ & \\
\hline More than $1,863.00$ USD & $382(0.5)$ & $12(4.6)$ & $370(5.0)$ & \\
\hline Don't know / Not informed & $11.66(15.3)$ & $33(12.7)$ & $1133(15.4)$ & \\
\hline Positive screening for a major depressive episode (N, \%) & $2201(28.8)$ & $120(46.3)^{*}$ & $2081(28.2)^{* *}$ & $<0.001^{\mathrm{a}}$ \\
\hline Positive screening for a bipolar spectrum disorder (N, \%) & $585(7.7)$ & $22(8.5)$ & $563(7.6)$ & 0.607 \\
\hline Positive screening for nicotine dependence (N, \%) & $459(6.0)$ & $33(12.7)^{*}$ & $426(5.8)^{* *}$ & $<0.001^{\mathrm{a}}$ \\
\hline Positive screening for alcoholism (N, \%) & $1759(23.0)$ & $82(31.7)^{*}$ & $1677(22.7)^{\text {** }}$ & $<0.001^{\mathrm{a}}$ \\
\hline Positive screening for suicidal ideation (N, \%) & $1909(25.0)$ & $107(41.3)^{*}$ & $1802(24.4)^{\star *}$ & $<0.001^{\mathrm{a}}$ \\
\hline a Pearson's chi-square test. & & & & \\
\hline${ }^{\mathrm{b}}$ Fisher's exact test. & & & & \\
\hline ' Two-tailed Student's $t$ test. & & & & \\
\hline${ }^{\mathrm{d}}$ Refers to an ethnic group of mixed white and black ance & & & & \\
\hline *0bserved was higher than expected in this cell (adjusted & & & & \\
\hline${ }^{* *}$ Observed was lower than expected in this cell (adjusted & & & & \\
\hline
\end{tabular}


TABLE 2. Psychopathological correlates of skin picking disorder (SPD)

\begin{tabular}{|c|c|c|c|c|}
\hline \multirow[b]{2}{*}{ Variable } & \multicolumn{2}{|c|}{ Unadjusted } & \multicolumn{2}{|c|}{ Adjusted $^{\mathrm{a}}$} \\
\hline & $\mathrm{OR}(95 \% \mathrm{Cl})$ & $P$-value ${ }^{d}$ & $\mathrm{OR}(95 \% \mathrm{Cl})$ & $P$-value ${ }^{\mathrm{d}}\left(\mathrm{R}^{2}\right)^{\mathrm{e}}$ \\
\hline Positive screening for a MDE (PHQ-9) & $2.198(1.714-2.820)$ & $<0.001$ & $1.854(1.430-2.403)$ & $<0.001(0.06)$ \\
\hline Positive screening for BSD (HCL-32) & $1.124(0.723-1.748)$ & 0.607 & $0.945(0.600-1.488)$ & $0.805(0.05)$ \\
\hline Positive screening for nicotine dependence (FTND) & $2.384(1.637-3.472)$ & $<0.001$ & $2.058(1.378-3.074)$ & $<0.001(0.05)$ \\
\hline Positive screening for alcoholism (AUDIT) & $1.575(1.206-2.057)$ & $<0.001$ & $1.570(1.193-2.065)$ & $0.001(0.05)$ \\
\hline \multicolumn{5}{|l|}{ Suicidality ${ }^{\mathrm{b}}$} \\
\hline Positive screening for suicidal ideation (PHQ-9) & $2.179(1.693-2.805)$ & $<0.001$ & $1.427(1.054-1.932)$ & $0.021(0.07)$ \\
\hline \multicolumn{5}{|l|}{ Psychopathological dimensions (SCL-90) ${ }^{c}$} \\
\hline Somatization & $1.186(0.942-1.493)$ & 0.147 & $1.085(0.855-1.376)$ & $0.502(0.1)$ \\
\hline Obsessive compulsive & $1.280(0.995-1.645)$ & 0.054 & $1.339(1.040-1.724)$ & 0.024 \\
\hline Interpersonal sensitivity & $0.780(0.603-1.009)$ & 0.059 & $0.759(0.583-0.989)$ & 0.041 \\
\hline Depression & $1.021(0.776-1.344)$ & 0.880 & $0.937(0.706-1.244)$ & 0.654 \\
\hline Anxiety & $1.242(0.888-1.737)$ & 0.205 & $1.154(0.821-1.624)$ & 0.409 \\
\hline Hostility & $1.291(1.069-1.560)$ & 0.008 & $1.300(1.074-1.573)$ & 0.007 \\
\hline Phobic anxiety & $0.936(0.760-1.154)$ & 0.538 & $0.919(0.745-1.135)$ & 0.434 \\
\hline Paranoid ideation & $1.142(0.897-1.454)$ & 0.282 & $1.191(0.928-1.528)$ & 0.171 \\
\hline Psychoticism & $1.162(0.869-1.553)$ & 0.311 & $1.322(0.978-1.788)$ & 0.069 \\
\hline \multicolumn{5}{|l|}{ Early-life trauma (ETISR-SF) $)^{b, c}$} \\
\hline General trauma & $1.093(1.029-1.161)$ & 0.004 & $1.070(1.005-1.139)$ & 0.034 \\
\hline Physical abuse & $1.022(0.927-1.126)$ & 0.663 & $1.078(0.974-1.194)$ & 0.147 \\
\hline Psychological abuse & $1.260(1.162-1.366)$ & $<0.001$ & $1.216(1.117-1.322)$ & $<0.001$ \\
\hline Sexual abuse & $1.081(1.007-1.160)$ & 0.032 & $1.042(0.968-1.121)$ & 0.278 \\
\hline
\end{tabular}

Abbreviations: AUDIT $=$ Alcohol Use Disorders Identification Test; BSD $=$ bipolar spectrum disorder; ETISR-SF $=$ Early Trauma Inventory Self Report - Short Form); FTND = Fagerström Test for Nicotine Dependence; HCL-32 $=$ Hypomania Checklist; $M D E=$ major depressive episode; PHQ $-9=$ Patient Health Questionnaire; RASS $=$ Risk Assessment Suicidality Scale; SCL-90 $=$ Symptom Checklist 90 .

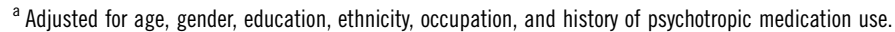

${ }^{\mathrm{b}}$ Adjusted additionally for a positive screening for MDD or BD, and tobacco or alcohol use disorder.

${ }^{\mathrm{C}}$ Per unity increase in dimension score.

${ }^{\mathrm{d}}$ Bold values are significant at a $5 \%$ alpha level after Bonferroni correction for multiple comparisons.

${ }^{\mathrm{e}}$ Nagelkerke $\mathrm{R}^{2}$ (ie, coefficients of determination) of each multivariable logistic regression model.

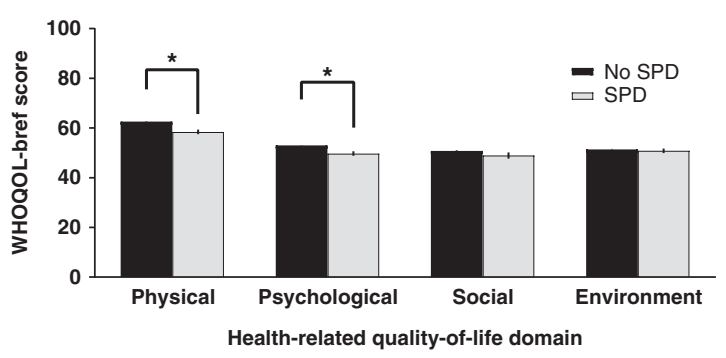

FIGURE 1. Associations between the presence of skin picking disorder (SPD) and physical, psychological, social, and environment quality of life as assessed with the WHOQOL-BREF. ${ }^{*} P<0.05$ (separate ANCOVA models adjusted for sociodemographic and psychopathological variables; see the Methods section for further details). Scores of WHOQOL-BREF domains are presented as means and $95 \% \mathrm{Cls}$.

clinical samples with SPD also pointed to high prevalence rates of co-occurring substance use disorders, including alcohol and tobacco use disorders. ${ }^{3,9,10}$ This finding is arguably consistent with a recent framework conceptualizing SPD as a "behavioral addiction," which may share some phenomenological and neurobiological similarities with substance use disorders. ${ }^{39}$
No significant association was found in our sample between SPD and a positive screening for bipolar spectrum disorder. Previously, a $10.5 \%$ prevalence of pathologic skin picking was reported in a sample of outpatients with bipolar disorder, ${ }^{40}$ but this investigation lacked a control (ie, comparison) group. A possible association between SPD and bipolar disorder does, however, deserve further investigation. In addition, we observed that SPD is associated with a positive screening for suicidal ideation that survived multivariable adjustment to potential confounders, including co-occurring affective disorders and alcohol use disorder, which are mental disorders associated with a higher suicide risk. ${ }^{41}$

As far as the SCL-90R is concerned, in our sample SPD was associated with the obsessive compulsive and hostility dimensions, with lower scores in the interpersonal sensitivity dimension. This finding suggests that SPD might share significant clinical and biological characteristics with other OCRDs, ${ }^{3,42}$ while the negative association with the interpersonal sensitivity dimension may be consistent with the view that OCRDs could be associated with anankastic personality features. ${ }^{43}$ However, it should be noted that a small study $(\mathrm{N}=92)$ indicated that 
participants with chronic skin picking exhibited elevated scores in experiential avoidance. ${ }^{10}$ Nevertheless, the small sample and the lack of a comparison group in that previous study may limit the generalizability of the findings. In addition, no previous study had specifically addressed the possible comorbidity of SPD with social anxiety disorder. In the current study, the presence of SPD was assessed by means of a reliable self-report measure. Yet the severity of SPD was not evaluated in this study. Thus, we cannot exclude the possibility that the association of SPD and interpersonal sensitivity could be moderated by the severity of SPD.

The association between SPD and exposure to childhood trauma (general traumas and psychological abuse) are consistent with other studies. ${ }^{14,15}$ However, the specificity and impact of a history of early life trauma remains to be established. It is noteworthy that other OCRDs (eg, OCD and trichotillomania) may also be associated with childhood trauma. ${ }^{43,44}$

\section{Associations of SPD and QoL}

Participants with SPD in our sample had significantly impaired physical and psychological QoL even after multivariable adjustment to sociodemographic and clinical variables. In addition, our data are consistent with available evidence that indicates SPD is associated with significant medical and psychological burden. ${ }^{3,13}$ Nevertheless, SPD was not associated with impaired social QoL. Although this finding is in agreement with the observation that participants with SPD exhibited lower scores in the interpersonal sensitivity dimension of the SCL-90R, it needs to be replicated, as it may also could be modified as a function of the severity of SPD. The instrument used in this survey (ie, the SPSQ) is primarily designed to screen for $\mathrm{SPD},{ }^{4}$ and not to rate the severity of this illness. Therefore, further research is warranted to assess the putative role of SPD's severity on the associations with QoL herein described.

\section{Strengths and limitations}

Some limitations of the current study deserve to be underlined. First, we enrolled a convenience Web-based sample with a predominance of young women that may not be representative of the Brazilian population. Second, although we used validated self-report measures in our study, a positive screening for SPD or other co-occurring disorders was not confirmed by means of a validated structured diagnostic interview. However, it should be noted that all instruments used in the current study exhibited adequate internal consistency reliabilities. Third, it is possible that this Web-based project attracted a greater proportion of participants with mental disorders, and hence the prevalence of SPD could have been over-estimated. Fourth, the cross-sectional design of the current study precludes the establishment of causal inferences. On the other hand, strengths of our study include the recruitment of a large sample and the employment of validated instruments with a widespread use in the scientific community. Furthermore, anonymous participation via Internet provides a setting with low desirability bias to answer to those instruments. This could be especially relevant in the case of OCRDs like $\mathrm{SPD}$, in which there is a long delay from the onset of symptoms to treatment initiation at least partly due to the "shame" individuals may experience due to their underlying condition. ${ }^{45}$

\section{Conclusions}

The current survey provides data to support the view that SPD could be a prevalent condition associated with significant comorbidities (namely depression, as well as tobacco and alcohol use disorders). In addition, SPD was associated with significantly impaired physical and psychological QoL even after adjustment for sociodemographic and clinical variables. Our findings may have some implications. First, efforts toward the early recognition of SPD (and associated comorbidities), particularly in at-risk settings (eg, dermatological clinics), seem to be warranted. In addition, future studies should investigate the determinants of QoL among people with SPD and consider using QoL as an outcome for therapeutic interventions targeting SPD.

\section{Disclosures}

Myrela Machado, Cristiano Köhler, Brendon Stubbs, Paulo Nunes-Neto, Ai Koyanagi, João Quevedo, Thomas Hyphantis, Donatella Marazziti, and Michael Maes have nothing to disclose. Jair Soares has the following disclosures: Pat Rutherford, Jr Endowed Chair in Psychiatry, personal fees; John S. Dunn Foundation, grant; National Institute of Mental Health, grant number R01MH085667-01A1; Bristol-Meyers Squibb, research support; Forest Laboratories, research support; Merck, research support; Elan Pharmaceuticals, research support; Johnson \& Johnson, research support; Stanley Research Institute, research support; National Institutes of Health, grant; Pfizer, served as a consultant; Abbott, served as a consultant; Astellas Pharma, served as a consultant. Dan Stein has the following disclosures: Lundbeck, personal fees; Novartis, personal fees; SUN, personal fees; CIPLA Inc., personal fees; AMBRF, personal fees; NRGF, grant; Servier, grant; Biocodex, grant; MRC, grant. André Carvalho reports grants from Conselho Nacional de Desenvolvimento Científico e Tecnológico $(\mathrm{CNPq})$ during the conduct of the study. 


\section{Supplementary materials}

To view supplementary material for this article, please visit https://doi.org/10.1017/S1092852918000871

\section{REFERENCES:}

1. Van Ameringen M, Patterson B, Simpson W. DSM-5 obsessivecompulsive and related disorders: clinical implications of new criteria. Depress Anxiety. 2014; 31(6): 487-493.

2. American Psychiatric Association. Diagnostic and Statistical Manual of Mental Disorders. 5th ed. Washington, DC: American Psychiatric Publishing; 2013.

3. Grant JE, Odlaug BL, Chamberlain SR, Keuthen NJ, Lochner C, Stein DJ. Skin picking disorder. Am J Psychiatry. 2012; 169(11): 1143-1149.

4. Keuthen NJ, Koran LM, Aboujaoude E, Large MD, Serpe RT. The prevalence of pathologic skin picking in US adults. Compr Psychiatry. 2010; 51(2): 183-186.

5. Hayes SL, Storch EA, Berlanga L. Skin picking behaviors: an examination of the prevalence and severity in a community sample. J Anxiety Disord. 2009; 23(3): 314-319.

6. Prochwicz K, Kałużna-Wielobób A, Kłosowska J. Skin picking in a non-clinical sample of young Polish adults. Prevalence and characteristics. Compr Psychiatry. 2016; 71: 77-85.

7. Siddiqui EU, Naeem SS, Naqvi H, Ahmed B. Prevalence of bodyfocused repetitive behaviors in three large medical colleges of Karachi: a cross-sectional study. BMC Res Notes. 2012; 5: 614.

8. Calikusu C, Kucukgoncu S, Tecer O, Bestepe E. Skin picking in Turkish students: prevalence, characteristics, and gender differences. Behav Modif. 2012; 36(1): 49-66.

9. Lochner C, Simeon D, Niehaus DJ, Stein DJ. Trichotillomania and skin-picking: a phenomenological comparison. Depress Anxiety. $2002 ; 15(2)$ : 83-86.

10. Flessner CA, Woods DW. Phenomenological characteristics, social problems, and the economic impact associated with chronic skin picking. Behav Modif. 2006; 30(6): 944-963.

11. Grant JE, Leppink EW, Tsai J, et al. Does comorbidity matter in body-focused repetitive behavior disorders? Ann Clin Psychiatry. 2016; 28(3): 175-181.

12. Grant JE, Menard W, Phillips KA. Pathological skin picking in individuals with body dysmorphic disorder. Gen Hosp Psychiatry. 2006; 28(6): 487-493.

13. Grant JE, Redden SA, Leppink EW, Odlaug BL, Chamberlain SR. Psychosocial dysfunction associated with skin picking disorder and trichotillomania. Psychiatry Res. 2016; 239: 68-71.

14. Favaro A, Ferrara S, Santonastaso P. Self-injurious behavior in a community sample of young women: relationship with childhood abuse and other types of self-damaging behaviors. J Clin Psychiatry. 2007; 68(1): 122-131.

15. Özten E, Sayar GH, Eryılmaz G, Kağan G, Işık S, Karamustafahığlu $\mathrm{O}$. The relationship of psychological trauma with trichotillomania and skin picking. Neuropsychiatr Dis Treat. 2015; 11: 1203-1210.

16. Lima AB, Köhler CA, Stubbs B, et al. An exploratory study of the heterogeneity of the jealousy phenomenon and its associations with affective temperaments and psychopathological dimensions in a large Brazilian sample. J Affect Disord. 2017; 212: 10-16.

17. Nunes-Neto PR, Köhler CA, Schuch FB, et al. Food addiction: prevalence, psychopathological correlates and associations with quality of life in a large sample. J Psychiatr Res. 2018; 96: 145-152.

18. Câmara RA, Köhler CA, Frey BN, Hyphantis TN, Carvalho AF. Validation of the Brazilian Portuguese version of the Premenstrual Symptoms Screening Tool (PSST) and association of PSST scores with health-related quality of life. Rev Bras Psiquiatr. 2017; 39(2): 140-146.

19. Lawshe CH. A quantitative approach to content validity. Personnel Psychology. 1975; 28(4): 563-575.

20. Angst J, Adolfsson R, Benazzi F, et al. The HCL-32: towards a self-assessment tool for hypomanic symptoms in outpatients. J Affect Disord. 2005; 88(2): 217-233.

21. Soares OT, Moreno DH, Moura EC, Angst J, Moreno RA. Reliability and validity of a Brazilian version of the Hypomania Checklist (HCL-32) compared to the Mood Disorder Questionnaire (MDQ). Rev Bras Psiquiatr. 2010; 32(4): 416-423.

22. Carvalho AF, Takwoingi Y, Sales PM, et al. Screening for bipolar spectrum disorders: a comprehensive meta-analysis of accuracy studies. J Affect Disord. 2015; 172: 337-346.

23. Santos IS, Tavares BF, Munhoz TN, et al. [Sensitivity and specificity of the Patient Health Questionnaire-9 (PHQ-9) among adults from the general population]. Cad Saude Publica. 2013; 29(8): 1533-1543.

24. Kroenke K, Spitzer RL, Williams JB. The PHQ-9: validity of a brief depression severity measure. J Gen Intern Med. 2001; 16(9): 606-613.

25. Choi YJ, Lee WY. The prevalence of suicidal ideation and depression among primary care patients and current management in South Korea. Int J Ment Health Syst. 2017; 11 : 18.

26. de Meneses-Gaya C, Zuardi AW, de Azevedo Marques JM, Souza RM, Loureiro SR, Crippa JA. Psychometric qualities of the Brazilian versions of the Fagerstrom Test for Nicotine Dependence and the Heaviness of Smoking Index. Nicotine Tob Res. 2009; 11(10): 1160-1165.

27. Heatherton TF, Kozlowski LT, Frecker RC, Fagerström KO. The Fagerstrom Test for Nicotine Dependence: a revision of the Fagerstrom Tolerance Questionnaire. Br J Addict. 1991; 86(9): 1119-1127.

28. Lima CT, Freire AC, Silva AP, Teixeira RM, Farrell M, Prince M. Concurrent and construct validity of the audit in an urban Brazilian sample. Alcohol Alcohol. 2005; 40(6): 584-589.

29. Saunders JB, Aasland OG, Babor TF, de la Fuente JR, Grant M. Development of the Alcohol Use Disorders Identification Test (AUDIT): WHO Collaborative Project on Early Detection of Persons with Harmful Alcohol Consumption-II. Addiction. 1993; 88(6): 791-804.

30. Osorio FL, Salum GA, Donadon MF, Forni-Dos-Santos L, Loureiro SR, Crippa JA. Psychometrics properties of early trauma inventory self report-short form (ETISR-SR) for the Brazilian context. PLoS One. 2013 ; 8(10): e76337.

31. Bremner JD, Bolus R, Mayer EA. Psychometric properties of the Early Trauma Inventory-Self Report. J Nerv Ment Dis. 2007; 195(3): 211-218.

32. Carissimi A. Examinando fatores causais de sintomas psicologicos atraves do SCL-90 R em pacientes com apneia do sono grave. Master's degree dissertation. Universidade Federal do Rio Grande do Sul, Porto Alegre, Brazil; 2011.

33. Derogatis LR, Melisaratos N. The Brief Symptom Inventory: an introductory report. Psychol Med. 1983; 13(3): 595-605.

34. Fleck MP, Louzada S, Xavier M, et al. [Application of the Portuguese version of the abbreviated instrument of quality life WHOQOL-bref]. Rev Saude Publica. 2000; 34(2): 178-183.

35. WHOQOL-Group. Development of the World Health Organization WHOQOL-BREF quality of life assessment. The WHOQOL Group. Psychol Med. 1998; 28(3): 551-558.

36. Cohen J. A power primer. Psychol Bull. 1992; 112(1): 155-159.

37. Monzani B, Rijsdijk F, Cherkas L, Harris J, Keuthen N, Mataix-Cols D. Prevalence and heritability of skin picking in an adult community 
sample: a twin study. Am J Med Genet B Neuropsychiatr Genet. 2012 159B(5): 605-610.

38. Leibovici V, Koran LM, Murad S, et al. Excoriation (skin-picking) disorder in adults: a cross-cultural survey of Israeli Jewish and Arab samples. Compr Psychiatry. 2015; 58: 102-107.

39. Chamberlain SR, Lochner C, Stein DJ, et al. Behavioural addiction-a rising tide? Eur Neuropsychopharmacol. 2016; 26(5): 841-855.

40. Karakus G, Tamam L. Impulse control disorder comorbidity among patients with bipolar I disorder. Compr Psychiatry. 2011; 52(4): 378-385.

41. Bolton JM, Gunnell D, Turecki G. Suicide risk assessment and intervention in people with mental illness. BMJ. 2015; 351: h4978.
42. Odlaug BL, Hampshire A, Chamberlain SR, Grant JE. Abnormal brain activation in excoriation (skin-picking) disorder: evidence from an executive planning fMRI study. Br J Psychiatry. 2016; 208(2): 168-174.

43. Stein DJ, Kogan CS, Atmaca M, et al. The classification of ObsessiveCompulsive and Related Disorders in the ICD-11. J Affect Disord. 2016; 190: 663-674.

44. Lochner C, Seedat S, du Toit PL, et al. Obsessive-compulsive disorder and trichotillomania: a phenomenological comparison. BMC Psychiatry. 2005; 5: 2.

45. Hollander E, Doernberg E, Shavitt R, et al. The cost and impact of compulsivity: a research perspective. Eur Neuropsychopharmacol. 2016; 26(5): 800-809. 